\title{
BMJ Open Management of patients with an advance decision and suicidal behaviour: a systematic review
}

\author{
Rebecca Nowland, ${ }^{1}$ Sarah Steeg, ${ }^{1}$ Leah M Quinlivan, ${ }^{1,2}$ Jayne Cooper, ${ }^{1}$ \\ Richard Huxtable, ${ }^{3}$ Keith Hawton, ${ }^{4}$ David Gunnell, ${ }^{5}$ Neil Allen, ${ }^{6}$ \\ Kevin Mackway-Jones, ${ }^{7}$ Navneet Kapur ${ }^{1,2,8}$
}

To cite: Nowland $R$, Steeg S, Quinlivan LM, et al. Management of patients with an advance decision and suicidal behaviour: a systematic review. BMJ Open 2019;9:e023978. doi:10.1136/ bmjopen-2018-023978

- Prepublication history and additional material for this paper are available online. To view these files, please visit the journal online (http://dx.doi. org/10.1136/bmjopen-2018023978).

Received 10 May 2018 Revised 19 November 2018 Accepted 28 November 2018

Check for updates

(C) Author(s) (or their employer(s)) 2019. Re-use permitted under CC BY. Published by BMJ.

For numbered affiliations see end of article.

Correspondence to Dr Rebecca Nowland; rnowland@uclan.ac.uk

\section{ABSTRACT}

Background The use of advance care planning and advance decisions for psychiatric care is growing. However, there is limited guidance on clinical management when a patient presents with suicidal behaviour and an advance decision and no systematic reviews of the extant literature.

Objectives To synthesise existing literature on the management of advance decisions and suicidal behaviour. Design A systematic search of seven bibliographic databases was conducted to identify studies relating to advance decisions and suicidal behaviour. Studies on terminal illness or end-of-life care were excluded to focus on the use of advance decisions in the context of suicidal behaviour. A textual synthesis of data was conducted, and themes were identified by using an adapted thematic framework analysis approach.

Results Overall 634 articles were identified, of which 35 were retained for full text screening. Fifteen relevant articles were identified following screening. Those articles pertained to actual clinical cases or fictional scenarios. Clinical practice and rationale for management decisions varied. Five themes were identified: (1) tension between patient autonomy and protecting a vulnerable person, (2) appropriateness of advance decisions for suicidal behaviour, (3) uncertainty about the application of legislation, (4) the length of time needed to consider all the evidence versus rapid decision-making for treatment and (5) importance of seeking support and sharing decisionmaking.

Conclusions Advance decisions present particular challenges for clinicians when associated with suicidal behaviour. Recommendations for practice and supervision for clinicians may help to reduce the variation in clinical practice.

\section{INTRODUCTION}

An advance decision (sometimes known as an advance decision to refuse treatment or living will) is typically a written document that outlines a person's desire to refuse certain treatments, including life-saving treatment, when there is a potential for a person to lose the mental capacity to make treatment decisions in the future. ${ }^{1}$ In order for an advance
Strengths and limitations of this study

- Timely systematic review considering the challenges relating to advance decisions in the context of suicidal behaviour.

- Review involves journal articles from a variety of countries from a range of disciplines.

- Paucity of evidence for this specific presentation of advance decision.

- Evidence in this area is predominately from reviews of case studies, rather than empirical work.

decision to be valid, the person must have mental capacity at the time of writing the document. Mental capacity is defined as the ability to make a decision and involves understanding and weighing information relating to a decision and alternative options and retaining that information long enough to make the decision. ${ }^{1}$ The Mental Capacity Act in England and Wales refers to 'advance decisions to refuse treatment', but more widely these documents are referred to as 'advance directives' and/or 'living wills'. We use 'advance decision' throughout in this paper to refer to written documents stating a refusal of treatment made in advance of medical treatment following an illness or injury.

There are important cross-national variations in legislation; in some countries, the use of advance decisions is not permitted (ie, Turkey, Japan), while in others, advance decisions are legislated for (ie, the UK and USA). The UK, Australia and USA have similar legal standards with some state-wide variation in the USA and Australia, ${ }^{2}$ with some states adopting the common law right to make an advance decision and others allowing the use of a surrogate or proxy decision-maker (ie, to make healthcare decisions on behalf of the patient). There is also considerable variation in practice between countries where advance decisions are permitted. For example, in 
Germany, advance decisions are recognised but require court approval in each case. ${ }^{2}$

Advance care planning for psychiatric care is becoming more common in a number of countries, including the UK, USA and Australia ${ }^{34}$ and enables patients to state their preferences for the management of their mental health when they may temporarily lose their mental capacity. A person with a mental health disorder may also make some decisions about particular treatment that they would not wish to have and may involve an advance decision to refuse particular treatments (ie, electroconvulsive therapy). Advance care planning has been shown to have a number of healthcare benefits for mental health patients in the UK and USA, such as enhancing patient autonomy and engagement, promoting adherence to treatment plans (ie, patients taking prescribed drugs), improving continuity of care with fewer psychiatric admissions, reducing the use of social workers' time and lower levels of violent acts. ${ }^{34}$ In a recent survey of patients with bipolar disorder, $21 \%$ had written statements about their healthcare, and of those, $10 \%$ involved an advance decision. ${ }^{5}$ This increasing use of advance care planning in mental health may result in an increasing use of advance decisions to refuse mental healthcare treatment, and concerns about clinical management of advance decisions following suicidal behaviour have been made by healthcare professionals and legal and ethical consultants. ${ }^{6-8}$ Existing literature, from a variety of academic and clinical perspectives, suggests there is little consistency in practice, and there are specific challenges with advance decisions following suicidal behaviour. Such scenarios raise questions about whether a person with a wish to end their life has the capacity to make a decision about refusal of treatment and/or if their capacity is affected by mental illness, and whether an advance decision is appropriate for medical treatment following suicidal behaviour. ${ }^{8}$

The terminology for suicidal behaviour varies internationally. Some clinicians/researchers distinguish between suicide attempts and non-suicidal self-injury, ${ }^{9}$ while others prefer the broad term of self-harm to denote behaviours across the spectrum. ${ }^{110}$ We have taken an inclusive approach in this review to ensure we captured relevant studies, so in this review we refer to 'suicidal behaviour' as behaviours including all self-harming behaviour (including non-suicidal injury) and suicide attempts. The use of 'suicidal behaviour' in our review means that there may be cases of non-suicidal injury that were included.

The management of suicidal behaviour is a significant challenge for clinicians in the emergency services. Each year over 200000 people present to emergency departments (EDs) in England with self-harm, ${ }^{10}$ with $16 \%$ of those presenting to hospital with a repeat selfharm episode within a year. ${ }^{11}$ Treatment refusal following suicidal behaviour has been shown to be common. A prospective cohort study of mental capacity and suicidal behaviour in the ED found that around $40 \%$ of patients presenting to hospital with self-harm had the capacity to make a decision about their medical treatment and 30\% of those intended to refuse life-saving treatment. ${ }^{12}$ There are few studies that have examined numbers of advance decisions to refuse treatment in patients presenting with suicidal behaviour, but in a recent study in three of 121 fatal cases of self-poisoning in 2005, patients had an advance decision. ${ }^{13}$ Given that patient autonomy and advance care planning are encouraged in modern healthcare and are assuming greater prominence, it is likely that the number of people presenting to hospital with an advance decision following suicidal behaviour will grow.

\section{Rationale}

While reviews of literature relating to the management of advance decisions, both more broadly and specifically to relating to 'end-of-life' care exist, ${ }^{1415}$ there are currently no reviews on the management of advance decisions when a patient presents to hospital following suicidal behaviour where the patient does not have a chronic or terminal physical illness. Despite the legislative context being similar for end-of-life care, the ethical considerations, emotional challenges and clinical decision-making may be different for treatment of a patient following suicidal behaviour without a chronic or terminal physical illness. A synthesis of this literature is important to examine similarities and differences and to establish the key findings. This is particularly important as the management of advance decisions to refuse treatment of injuries and illnesses following suicidal behaviour is challenging for clinicians $^{8}$ and there is a lack of consistency of practice. A review of the literature will be important to inform guidelines for the management of advance decisions following suicidal behaviour.

\section{Aim}

To systematically review and synthesise literature on the treatment and clinical management of patients presenting to hospital with an advance decision to refuse treatment following suicidal behaviour without a chronic or terminal physical illness. The review was conducted by researchers in the UK, but an examination of all the existing literature was conducted without language or country restrictions.

\section{METHOD}

The review was conducted in accordance with Preferred Reporting Items for Systematic Reviews and Meta-Analyses (PRISMA) guidelines ${ }^{16} 17$ and guidance for conducting narrative synthesis in healthcare. ${ }^{18}$ There is no protocol for the review. We used the PRISMA checklist when writing our report. ${ }^{16}$

\section{Search strategy and data sources}

An initial scoping of the literature was conducted at inception of the study and the findings were used to inform the search strategy. Content experts and clinical practitioners on the research team assisted with compiling keywords and/or phrases (see table 1). In order to take 
Table 1 Search terms for each topic

\begin{tabular}{|c|c|c|c|c|}
\hline Advance directives & OR & Mental capacity & AND & Suicidal behaviour \\
\hline $\begin{array}{l}\text { advance decisions } \\
\text { advance directives } \\
\text { advance statement } \\
\text { living will(s) } \\
\text { mental health directive } \\
\text { Ulysses contract(s) } \\
\text { psychiatric will(s) } \\
\text { antecedent decision/wish } \\
\text { pre-emptive suicide } \\
\text { antecedent refusal } \\
\text { resuscitation order } \\
\text { health care power of attorney }\end{array}$ & & $\begin{array}{l}\text { mental competency } \\
\text { mental capacity }\end{array}$ & & $\begin{array}{l}\text { suicide } \\
\text { attempted suicide } \\
\text { self-mutilation } \\
\text { self-harm } \\
\text { deliberate self-harm } \\
\text { parasuicide } \\
\text { self-injurious behaviour } \\
\text { drug overdose } \\
\text { self-immolation } \\
\text { self-poisoning } \\
\text { self-destructive behaviour } \\
\text { auto aggression } \\
\text { automutilation }\end{array}$ \\
\hline
\end{tabular}

an inclusive approach and enable inclusion of any papers that involved discussion of management of advance decisions following 'suicidal behaviour' we included a variety of key search terms relating to non-accidental injury and suicide attempts. An electronic search of six databases (EMBASE, MEDLINE, PSYCHINFO, Social Policy and Practice, CINAHL and Medline) was conducted, as well as a full electronic search on WestLaw (an online library of UK legal information) using the following search terms: advance decisions, advance directives AND wills, suicide. Full search strategy for each database is supplied as supplementary information (online supplementary information $1)$. In addition, the reference sections of all included sources were consulted and authors' personal files were also searched to ensure that potentially eligible sources were not omitted. No study design, date or language restrictions were imposed.
Literature searches were conducted during the period April 2016-July 2018. The specific inclusion and exclusion criteria are detailed in table 2.

\section{Study selection}

Titles and abstracts were screened, with a random sample of $10 \%$ of the articles independently screened by another researcher. Additional information was sought where there were any disagreements, which were then resolved through discussion. An acceptable concordance rate between the inclusion decisions was predefined as agreement on at least $90 \%$ of the articles, which was achieved for screening on title and abstract. Full text screening of the selected articles was conducted by two researchers independently, with full agreement being achieved at this stage.

Table 2 Criteria for inclusion and exclusion

\begin{tabular}{|c|c|c|}
\hline Parameter & Inclusion criteria & Exclusion criteria \\
\hline Patients & $\begin{array}{l}\text { Patients over } 18 \text { years who present to hospital with advance } \\
\text { decisions* (also include do not resuscitate orders, DNRs) } \\
\text { following suicidal behaviour (including attempted suicide, } \\
\text { deliberate self-harm, self-injurious behaviour, drug overdose, } \\
\text { self-poisoning, self-destructive behaviour) with no existing } \\
\text { chronic or terminal physical conditions. }\end{array}$ & $\begin{array}{l}\text { Patients who present to hospital with } \\
\text { advance decisions but with primary } \\
\text { conditions which were not mental } \\
\text { health related (eg, HIV/AIDS, chronic } \\
\text { physical health conditions or disabilities, } \\
\text { neurodegenerative diseases and/or specific } \\
\text { patient groups (eg, mother/baby)). }\end{array}$ \\
\hline
\end{tabular}

Comparator

Outcomes Adherence/non-adherence with advance decision, treatment, patient outcome (ie, death).

Study design Opinion and review articles, case studies, empirical studies/ surveys.

Book reviews, responses to articles, conference abstracts.

*Or other terms such as advance decisions, advance directives, advance statement, living will(s), mental health directive, Ulysses contract(s), psychiatric will(s), mental competency, mental capacity, healthcare power of attorney, antecedent decision/wish, pre-emptive suicide, antecedent refusal, resuscitation order or living will, advance directive, Ulysses contract. 


\section{Data extraction and analysis}

A preliminary analysis of the data was conducted. ${ }^{18}$ Studies were from a range of disciplines (ie, general medical, psychiatry, ethical, legal) and involved reviews of clinical cases or fictional scenarios. It was deemed appropriate to conduct a narrative synthesis because this particular approach is useful when synthesising textual findings from diverse literatures. ${ }^{18}$ Narrative synthesis was conducted in two phases: (1) a textual synthesis and (2) an adapted thematic framework analysis. ${ }^{19}$

First, the textual synthesis of the data was conducted by extracting key factual information from each study (country of origin, perspective/discipline, factual or fictional case study) and details of the case studies (age of patient, mental health disorders, nature of suicidal behaviour, resulting injuries/illness, hospital admittance, type of advance decision, when the advance decision was written, and whether patient was conscious, decision-making processes). The information was then summarised and tabulated to map the literature that cited the same clinical case. Information from cases only involving a factual case study (ie, a real clinical case) was extracted because we were interested in information about actual clinical cases, decision-making process and rationale for decisions made. Thus, information was not extracted from reports that discussed a hypothetical scenario for the textual synthesis. Data extraction and summarisation was completed independently by two researchers using a predetermined data extraction sheet.

Second, an adapted thematic framework analysis approach $^{19}$ was used to examine key themes discussed in the selected papers. This involved five stages: initial open coding, indexing, descriptive summaries, charting and tabulation and interpretation. Initial open coding generated three general categories representing the most discussed issues across the selected articles: (1) key issues with an advance decision relating to suicidal behaviour, (2) challenges in clinical decision-making for advance decisions relating to suicidal behaviour and (3) recommendations for practice. These three categories were used to index the data and as a framework to extract and summarise data. Extracted data were then used to form descriptive summaries. Indexing, extracting and summarising were conducted independently by two researchers. Resulting summaries were compared and discussions were held to clarify any differences. Charting and tabulation was conducted by charting the summaries by discipline. In order to explore similarities and differences between disciplines, we distinguished between 'General Medical' as papers written from a general medical practice or emergency services perspective; 'Psychiatry' as those written by clinical psychiatrists or from a psychiatry perspective, 'Nursing' as those written by practising nurses or research nurses, 'Bioethics' as those in ethics sections in journals or written by researchers in medical ethics, 'Ethics' as those in ethics journals or written by ethics researchers and 'Legal' as those written from a legal perspective and/or by a legal representative. Interpretation of the data was conducted by thematic analysis of the summary charts to highlight the main recurrent and most important themes. ${ }^{18}$ Two researchers conducted the thematic analysis independently and then discussed and finalised themes. Saturation of the themes was established when no further themes emerged and could not be further collapsed. 'Vote counting' was used to identify the frequency with which the themes appeared in the selected papers. ${ }^{20}$ In the thematic framework analysis all selected studies were included; those involving a factual case and those involving a fictional case, because both involved discussions of concerns, challenges and rationale for decision-making relating to management of an advance decision following suicidal behaviour.

\section{Quality assessment}

The papers mostly comprised accounts of clinical cases written by clinicians and ethical or legal experts. The methodology quality and synthesis of case series and case reports tool suggested by Murad and colleagues ${ }^{21}$ was used to assess the quality of selected studies. Each study was assessed independently across four areas of potential bias: selection, ascertainment, causality and reporting. The tool consisted of five items each requiring a binary response to indicate whether the bias was likely. We considered the quality of the study good when all five criteria were fulfilled, moderate when four were fulfilled and poor when three or fewer were fulfilled. The methodological quality of included studies was assessed independently by two reviewers and discussions were held between them where there was disagreement. We also considered the reflexivity of the author/s, their expertise and how they were involved in the clinical case (eg, as a clinician or legal/ethics consultant). Authors of the papers reflected on the management of the clinical case, rationale for decision made and issues relating to advance decisions and suicidal behaviour more generally.

\section{Patient and public involvement}

An expert-by-experience was a co-applicant on the National Institute of Health Research (NIHR) Programme Grant and actively contributed to the study design and objectives. Patient advisors, carers and clinicians evaluated the relevance and importance of the research questions for the advance decisions component of the grant and the systematic review. Our interim and final results were presented and evaluated by clinicians, academics, patients and carers. There was also patient input into our dissemination plan which includes dissemination to clinicians and the relevant patient community.

\section{RESULTS}

\section{Systematic search}

Results of the systematic search are displayed in figure 1 . After duplicates were removed, the search returned 634 articles, of which 35 were retained after screening based 


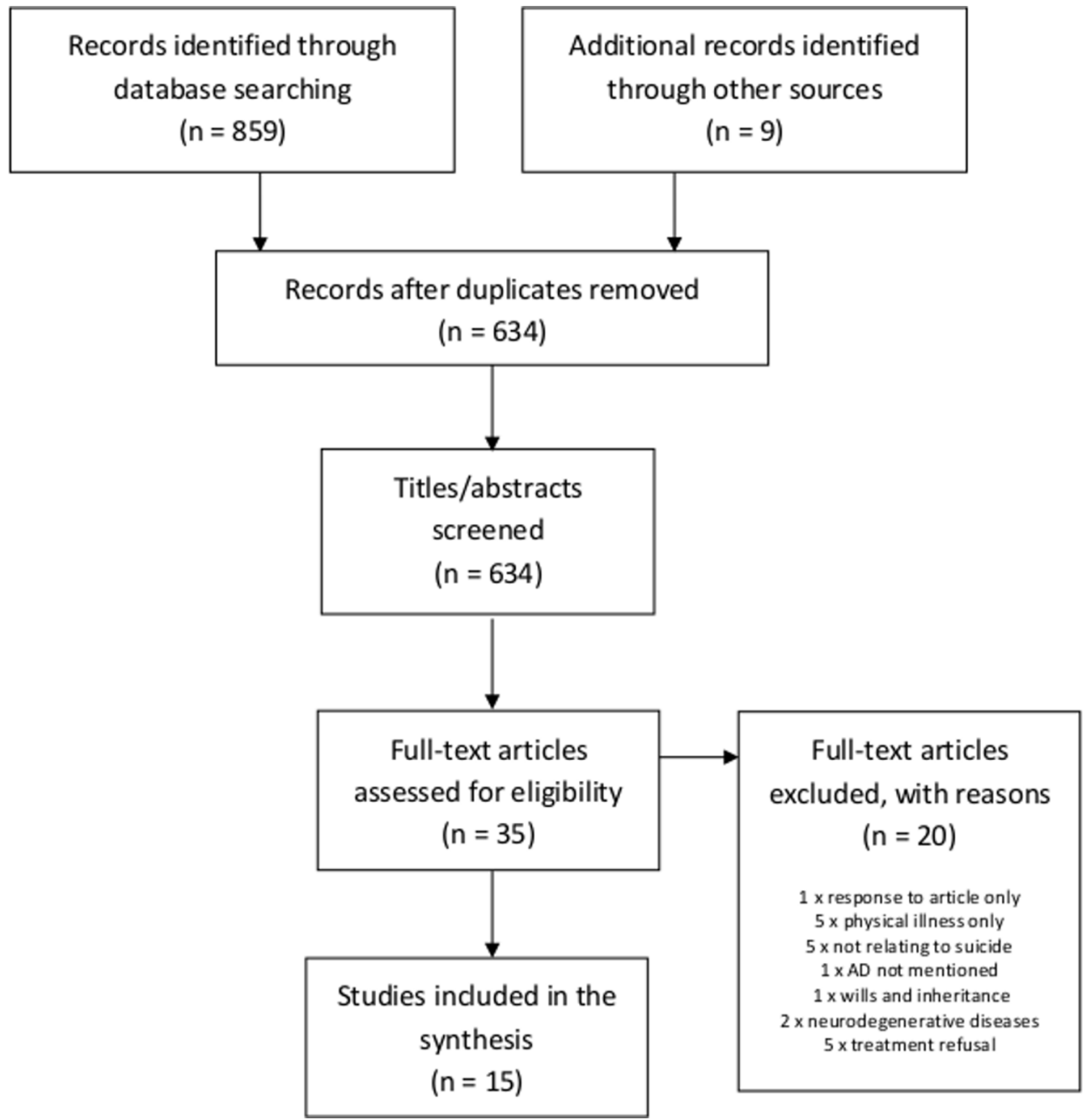

Figure 1 Flow chart of results from initial search. AD, advance decision.

on title/abstract. Following full-text screening, 15 articles were retained for data extraction.

\section{Study characteristics}

Descriptive information about the selected articles is displayed in table 3. Five of the selected articles were from the UK and the others were from the USA $(n=7)$ or Australia $(n=3)$. A total of six clinical cases were reviewed across the 15 articles (see table 3 ), as seven (47\%) of the articles reported the same case (case A, a well-publicised case of a 26-year-old woman who died in the UK). Two of the clinical cases presented fictional scenarios. ${ }^{22}$

\section{Study quality assessment}

All 15 studies were assessed for bias using the methodology quality and synthesis of case series and case reports tool suggested by Murad and colleagues. ${ }^{21}$ Nine of the selected studies were deemed to have moderate methodological quality and six to have poor quality (see online supplementary information 2). The quality assessment is supplied as supplementary information (online supplementary information 2). None of the studies reported the representativeness or selection process relating to the case report, which impacted on the bias ratings. Although case reports are considered to have increased risk of bias, they have profoundly influenced medical literature and advanced knowledge and their use in reviews is considered appropriate where no other higher level evidence is available. $^{21}$

\section{Textual synthesis}

Examination of clinical cases discussed in the selected articles Specific information about clinical cases and decision-making is summarised and charted in table 4 . We only included examination of the factual cases $(n=6)$ in this part of the analysis, because we were interested in the types of real-world cases and decisions made, rather than an examination of a hypothetical scenario.

Patients discussed in the clinical cases varied in age, ranging from 26 to 86 years old. All patients were noted as having a diagnosis of depression, some were reported as also having diagnoses of post-traumatic stress disorder and personality disorders. The suicide methods used in the cases included self-poisoning $(n=3)$, gunshot incidents $(n=2)$ and hanging $(n=1)$. All patients were found by other people, except one patient who called an ambulance because they did not want to die alone. Four of the 
Table 3 Description of selected studies

\begin{tabular}{|c|c|c|c|c|c|}
\hline Author & Date & Country & Perspective* & $\begin{array}{l}\text { Fictional/factual } \\
\text { case }\end{array}$ & Case reported $\dagger$ \\
\hline Bryne $^{2}$ & 2002 & Australia & Nursing & Fictional & - \\
\hline Callaghan and Ryan ${ }^{26}$ & 2011 & Australia & Bioethics & Factual & $A$ \\
\hline Chalfin et $\mathrm{al}^{25}$ & 2001 & $\begin{array}{l}\text { USA, Philadelphia, } \\
\text { New York, New } \\
\text { Zealand }\end{array}$ & $\begin{array}{l}\text { Emergency and acute } \\
\text { medicine/bioethics }\end{array}$ & Factual & B \\
\hline Dresser $^{6}$ & 2010 & USA, New York & Legal & Factual & $A$ \\
\hline David et $a l^{27}$ & 2010 & UK & Psychiatry & Factual & $A$ \\
\hline Frank $^{7}$ & 2013 & USA, Colorado & Legal & Factual & $\mathrm{D}$ \\
\hline Kapur et $a l^{8}$ & 2010 & UK & Psychiatry & Factual & $E$ \\
\hline Mitchell ${ }^{22}$ & 2011 & USA, San Diego & Ethical & Fictional & - \\
\hline Ryan and Callaghan ${ }^{30}$ & 2010 & Australia & Psychiatry & Factual & A \\
\hline Sontheimer ${ }^{24}$ & 2008 & USA, Springfield & Bioethics & Factual & $E$ \\
\hline Szawarski $i^{31}$ & 2013 & UK & Bioethics & Factual & $A$ \\
\hline Volpe et $\left.a\right|^{32}$ & 2012 & USA, New York & Bioethics & Factual & $\mathrm{F}$ \\
\hline
\end{tabular}

*Where the perspective is not clearly stated, this has been derived from the author(s) background and professional experience. †For specific details about each case, see table 4. Note: fictional cases have not been given a case report ID.

patients were reported to have died; the outcome in one case was not specified.

Treatment was provided in only one of the clinical scenarios. ${ }^{23}$ In this case, the patient was a psychiatric inpatient and the advance decision was considered part of the suicide attempt, so the patient's treatment refusal specified in the advance decision document was not adhered to.

The rationale for non-treatment in the clinical cases where the patient died varied and was summarised into the following three reasons:

- Advance decision was followed as a legally-binding document after checks showed the information was clear and specific, patient was informed of treatment options, had mental capacity at the time of writing and family were in agreement with the decision for non-treatment $(n=1) .{ }^{8} 24$

- Physical injuries were severe resulting in poor prognosis for the patient and the treatment refusal in the advance decision was used as evidence that the patient would not wish to survive with a life-threatening or severely disabling condition. Where possible, families were also consulted $(n=2) .^{725}$

- Verbal treatment refusal was used as the basis for the treatment decision, rather than the advance decision, because the patient was conscious and had mental capacity. Consultation with family was not reported in this case. $(n=1) .^{626-31}$

The decision-making process was reported to take considerable time and legal and/or ethical consultation took place in all the reported clinical cases.
Differences in opinions about clinical management and decision-making between ED clinicians and psychiatric consultants were reported in some of the clinical cases. $^{2325}$ In those cases, ED clinicians gave more weight to the advance decision, suggesting it should be adhered to as a legally binding document and the patient remain untreated. In contrast psychiatrists viewed suicide as a consequence of a distressed state and expressed a preference to avoid adherence with the advance decision and treat the patient. Where such conflict arose this was resolved through consultation with the hospital legal team and/or ethics committee.

\section{Thematic analysis}

Five themes arose from the thematic analysis and are presented with their corresponding subthemes and votecounts in table 5 . We included accounts of fictional cases in the thematic analysis because here we were interested in opinions, views and perspectives of authors.

\section{Themes}

Tension between patient autonomy and protecting a vulnerable person

Professional dilemma: promoting patient autonomy versus providing appropriate care

The management of an advance decision in the context of suicidal behaviour was particularly challenging because it went against healthcare professionals' training to preserve life (ie, adherence to the advance decision could result in the death of the patient while they could recover if they received treatment for their physical condition). This 


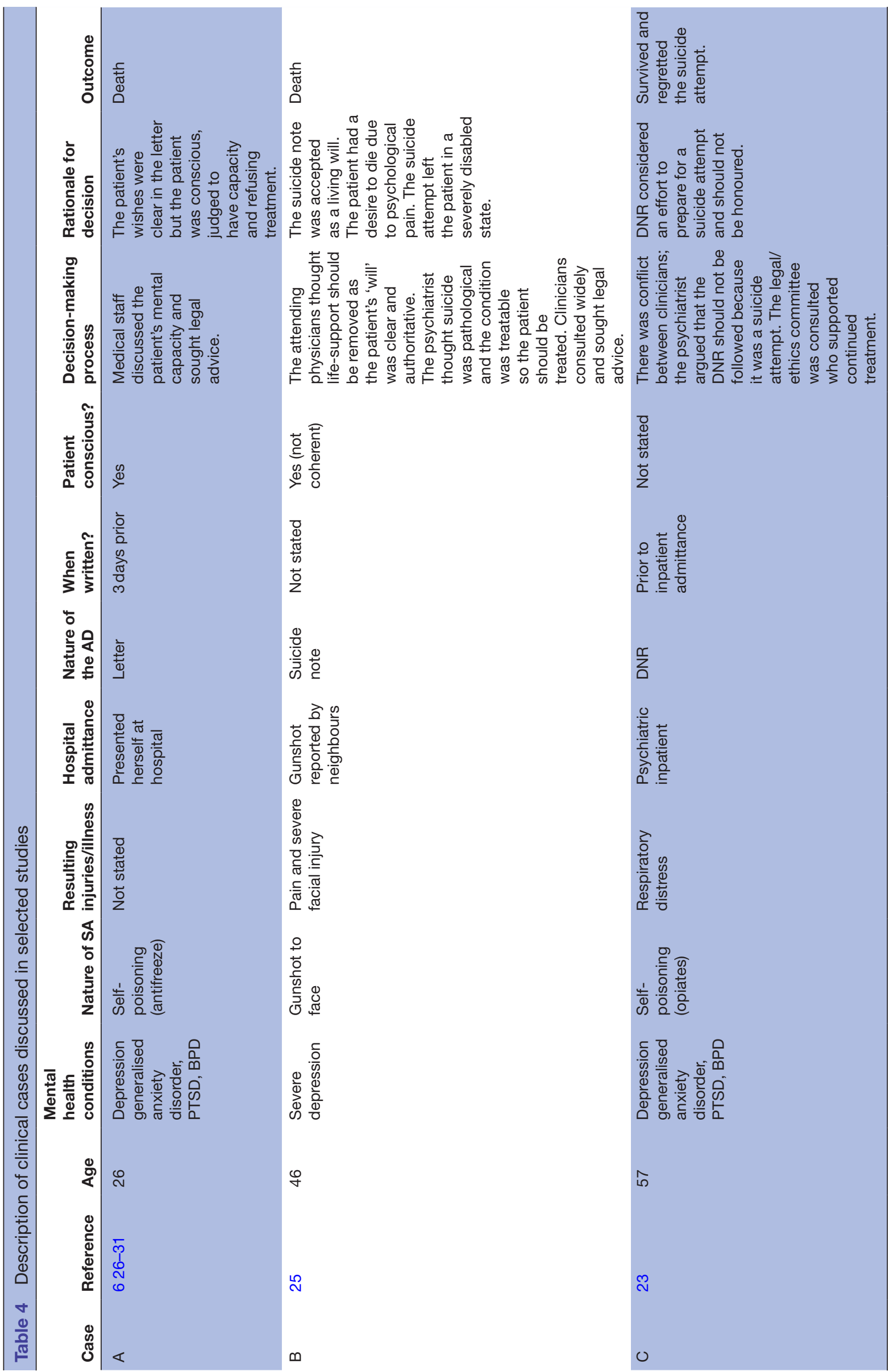




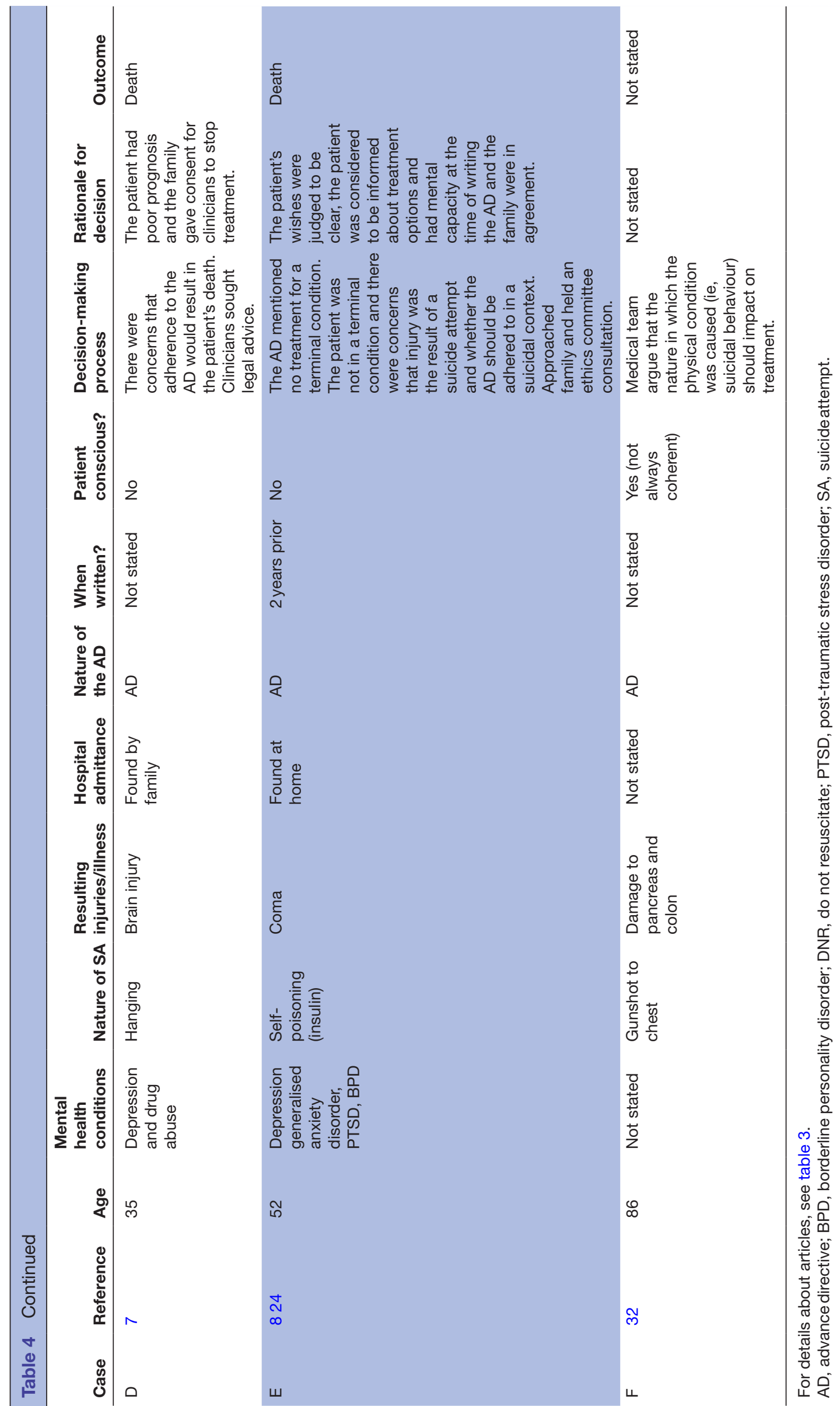




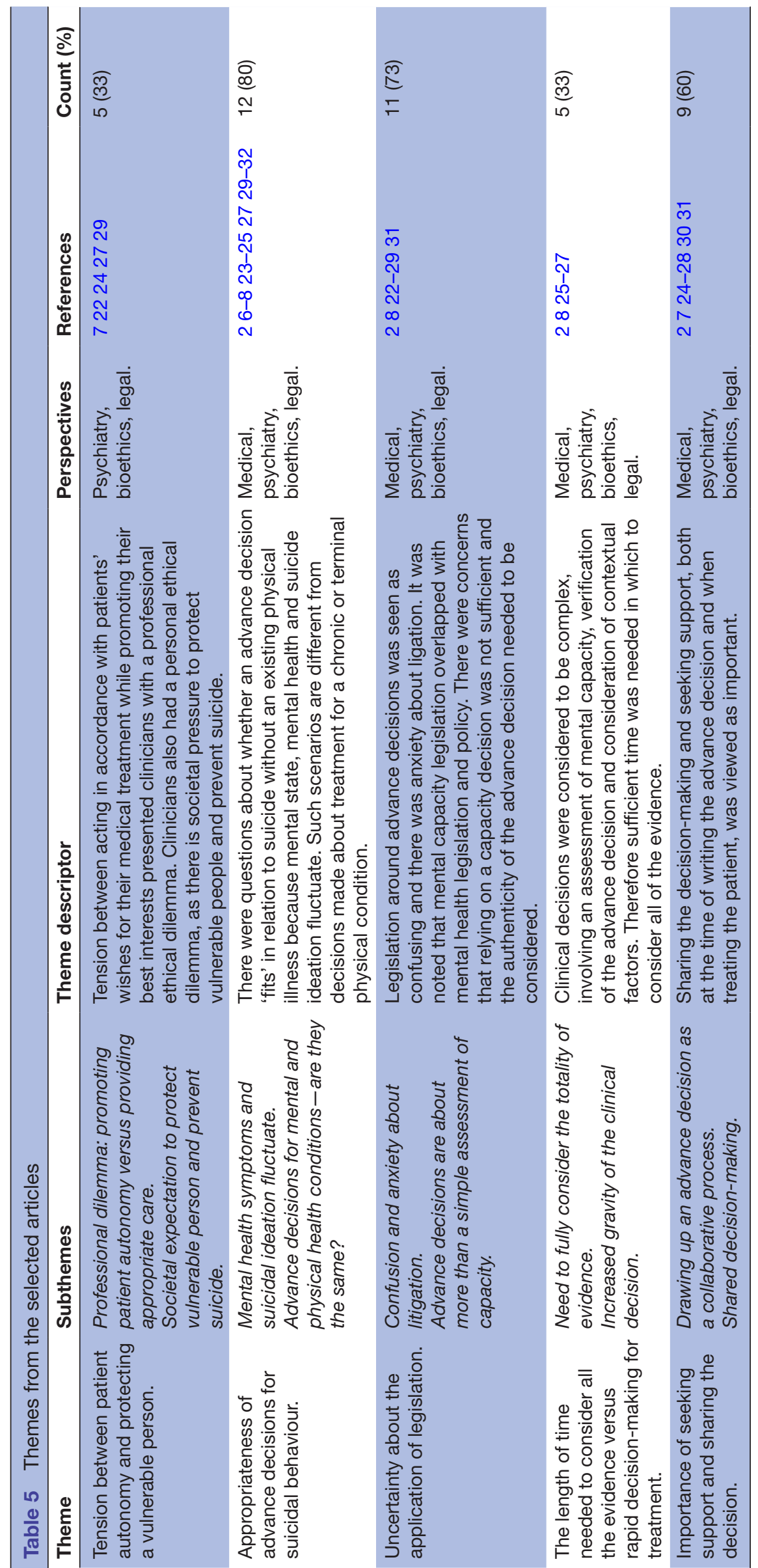


presented clinicians with a dilemma between promoting patients' autonomy by observing their wishes stated in the advance decision and by providing care that was considered in their best interests (eg, promoting life). ${ }^{723262830}$

\section{Societal expectation to protect vulnerable person and prevent suicide}

Authors also raised the issue that clinicians not only had a professional interest in protecting a vulnerable person, but there was also a societal expectation that suicide should be prevented. ${ }^{23} 2530$

While the right to autonomy is strong, in some circumstances there may be competing rights and interests that are sufficient to override a competent decision to refuse treatment. These may include the state's interests in preventing suicide. ${ }^{30}$

The challenge to clinicians was highlighted by an acknowledgement from some authors that adherence to the advance decision in this context was emotive and would feel like assisting suicide. ${ }^{24} 30$

\section{Appropriateness of advance decisions for suicidal behaviour} Mental health symptoms and suicidal ideation fluctuate

Concerns were expressed about whether an advance decision should apply in the context of suicidal behaviour because of the patients' distressed state, the potential for suicidal ideation to fluctuate and for treatment preferences to change in the future. ${ }^{783132}$

The compelling notion that people will change their minds contradicts the primacy of patient autonomy in the consideration of suicide. This is what distinguishes an impulsive suicide attempt from other informed choices to obtain or refuse medical treatment by patients. ${ }^{7}$

Authors from a psychiatric perspective, in particular, viewed suicidal behaviour as a symptom of a mental health disorder that was potentially treatable with psychiatric care. ${ }^{25}$ They also expressed concerns about the capacity of a distressed suicidal person to fully comprehend their decision and consider all treatment options available to them. $^{2} 242532$ Therefore, it was suggested by some authors that a higher level of mental capacity may be required at the time of writing the advance decision for clinicians to be confident in following it. ${ }^{8}$ However, other authors argued that the advance decision should be considered as part of the suicide attempt and as evidence of distressed/ disordered thinking, ${ }^{8327} 28$ rather than independently of the attempt and the treatment refusal in the advance decision document should not be adhered to.

\section{Advance decisions for mental and physical health conditions - are} they the same?

The difference between an advance decision for suicidal behaviour and for a physical condition was highlighted across the selected papers. ${ }^{6}{ }^{32}$ Authors from a legal perspective highlighted that the primary aim of an advance decision relating to a suicide attempt is to end life, whereas an advance decision for a chronic or terminal illness is often concerned with managing pain and avoiding prolonged suffering. ${ }^{6}$

There was also debate about the extent to which mental suffering legitimised suicide. ${ }^{32}$ Authors from an ethical perspective argued that, typically, healthcare services may be more sympathetic to 'end-of-life' decisions relating to terminal physical health conditions than mental health conditions, thus mental health patients do not receive the same palliative care options as patients without mental health diagnoses. ${ }^{24}$ There was some discussion that it should not be assumed that psychiatric pain is more tolerable than physical pain and that both should be considered as having a similar influence on the patient. ${ }^{24} 25$

Uncertainty about the application of legislation Confusion and anxiety about litigation

Authors from general medical and psychiatry perspectives expressed confusion about legislation and anxiety about litigation, ${ }^{2} 230$ with one stating that the advance decision document needed to be 'watertight' to be considered. ${ }^{25}$ Authors recommended that clear hospital policies be developed for advance decisions in this particular context to overcome the confusion and anxiety about ligation. ${ }^{23}$

In addition to the clinical demands associated with treating a patient with a life-threatening condition, clinicians must do their best to ascertain the patient's capacity for his or her apparent decision, consider the correct ethical course, and navigate through uncharted legal waters. ${ }^{7}$

Authors from the UK and Australia highlighted the difficulties in implementing both mental health and mental capacity legislation when managing advance decisions relating to suicidal behaviour. ${ }^{27}{ }^{29-31}$ Clinicians needed to consider whether someone who had attempted suicide was suffering with a mental health condition, for which they should be treated against their will. They also needed to judge whether the person had the capacity to make a decision about their treatment and, if so, that the advance decision could apply following verification checks. Some suggested that application of each legislation model (ie, mental health or mental capacity), in isolation of the other, could result in different outcomes for the patient. ${ }^{6}$ Some authors suggested that the difficulty with balancing mental capacity legislation and mental health legislation could be resolved by developing a single legislation that combines both. ${ }^{827}$

\section{Advance decisions are about more than a simple assessment of capacity}

A reliance on judging a person's capacity to make a decision in the context of suicidal behaviour was discussed in detail. ${ }^{822} 24$ The capacity assessment was discussed in relation to when the patient was involved in advance care planning and making the decision to write an advance decision to refuse treatment. ${ }^{8}$ Capacity assessment was 
also discussed in relation to clinicians in an emergency situation, treating a person who is considered to have capacity to verbally refuse or accept treatment. In this scenario the advance decision can be ignored. While this is an important part of some legislation, particularly in the UK, it was suggested that an assessment of capacity should be supplemented with a judgement of the authenticity and durability of the patient's decision (ie, if the decision had been consistent over time). ${ }^{22}{ }^{26}$ Authors from a psychiatric perspective, in particular, suggested that advance decisions should be regularly reviewed to ensure that they were up-to-date and continued to reflect the patient's desires and preferences. ${ }^{26-28}$

The length of time needed to consider all the evidence versus rapid decision-making for treatment

\section{Need to fully consider the totality of evidence}

Some authors suggested that the increased length of time taken in this particular context arose from the need to consider contextual factors for the suicidal behaviour, 2225 the patient's mental health background ${ }^{27}$ and the reason for their decision, alongside the usual validation checks and judgement of the presence of mental capacity at the time of making the advance decision. It was also argued that clinicians should take into account wider factors that may have not been present when the person first wrote the advance decision, such as changes in evidence for a particular treatment or scientific advances offering new treatment options that may influence the patient's decision. ${ }^{22}$

However, authors highlighted difficulties with gaining access to such evidence, particularly in emergency situations, further adding to the time taken to make a decision. ${ }^{31}$ It was noted that advance decisions were often too specific (eg, related to a specific illness or injury) or too general (eg, a general refusal of treatment, rather than refusal of a specific treatment), resulting in ambiguity as to the best course of action for the patient and time consuming investigation. ${ }^{2528}$ Some authors highlighted that advance decisions were not useful in emergency settings when rapid decision-making was required ${ }^{2}$ but may be appropriate for patients to express refusals of on-going psychiatric treatment (eg, electroconvulsive therapy).

\section{Increased gravity of the clinical decision}

Authors argued that the gravity of the clinical decision was increased in this context because the patient could die if the advance decision was adhered to when recovery from mental ill health may be possible. ${ }^{625}$ Authors suggested that validation checks in this context may need to be more thorough and authors from a legal perspective argued that, because of the increased gravity of the clinical decision, physicians should seek a consensus about clinical management, while providing life-sustaining treatment, creating a time-consuming situation. ${ }^{731}$

Importance of seeking support and sharing the decision Drawing up an advance decision as a collaborative process

Someauthorsargued thatwhen writinganadvancedecision, patients should be supported by a healthcare professional to consider all possible treatment options. ${ }^{22} 232729$ It was suggested that evidence of mental capacity at the time of writing the advance decision should be provided (eg, verified and signed by the healthcare professional) which could help with clinical decision-making at a later stage. ${ }^{22}$ Authors from all the perspectives stressed the importance of also consulting with a physician at the time of writing the advance decision to ensure that it is both specific and general enough to be helpful and informative in a given medical scenario. ${ }^{2327}$

\section{Shared decision-making}

All authors discussed the need for multiagency decision-making in relation to the management of advance decisions in the context of suicidal behaviour. ${ }^{72728}$ Suggestions included that clinicians should consult widely, make use of psychiatric expertise, review the patient's psychiatric history and background and seek legal and/or ethical consultation when considering treatment decisions.

\section{DISCUSSION}

\section{Summary of the findings}

A comprehensive systematic review of studies examining the management of advance decisions to refuse treatment following suicidal behaviour was conducted. The findings show a paucity of studies in this specific area. Fifteen relevant studies were identified, of which all were reports of clinical cases. With the exception of two papers that noted fictional clinical cases, the others reported on six real clinical cases. Despite having no language or country restrictions to the search, all the studies were from the USA, Australia or UK which have similar legislation relating to advance care planning and advance decisions to refuse treatment. ${ }^{2}$

There were inconsistent views on practice and rationales for the management of advance decisions. Treatment was provided in only one clinical case, where the patient was a psychiatric inpatient and the advance decision was considered part of the suicide attempt. ${ }^{23}$ In this case the patient survived and later regretted the suicide attempt. In the other clinical cases, treatment was not provided, but rationale for non-treatment differed. Rationale for treatment varied from viewing the advance decision as legally binding ${ }^{824}$ to using the advance decision as an aide to understand the patients' treatment preferences when there was a poor prognosis or a resulting severely disabling condition. ${ }^{725}$

Conflict between clinicians was reported in some of the cases. ${ }^{23}{ }^{25}$ In the studies where there were conflicts, there were differences in opinions on treatment between ED clinicians and psychiatrists. Consultations with mental healthcare staff were typically sought when a patient presented with an advance decision following suicidal behaviour. Psychiatrists tended to stress the treatable nature of a mental health condition and that the suicidal behaviour was part of the mental health disorder. In contrast, ED clinicians argued that the advance decision 
document was legally binding and expressed anxieties about litigation. These differences in opinion about treatment were overcome through consultations with legal and ethical representatives.

The appropriateness of advance decisions with suicidal behaviour was questioned for two reasons. First, suicide ideation was considered to fluctuate and people could change their mind about their desire to die. ${ }^{783132}$ Although suicide has been linked to impulsivity, ${ }^{334}$ studies show that not all suicides are impulsive. ${ }^{35}$ However, recent studies using ecological momentary assessment have shown that suicide ideation varies over short periods of time (ie, there are changes between hours and days) ${ }^{36}$ and follow-up studies with suicide survivors tend to acknowledge that they regret the suicide attempt. ${ }^{37}$ Second, outcomes for treatment refusal following suicidal behaviour were noted to be potentially different to those for a terminal physical health condition (ie, the patient could die when there is potential for recovery in the future) ${ }^{632}$

Authors discussed concerns that management of advance decisions following suicidal behaviour may need to be different because they are a unique clinical presentation. Similar to findings in this review, anxieties and confusion about legislation relating to advance decisions is also found in studies examining end-of-life care. ${ }^{38}$ However, what differs is opinions about adherence to the advance decision to refuse treatment for chronic or terminal conditions and sympathy for assisted suicide in end-of-life care. Healthcare workers report support for assisted suicide relating to end-of-life care ${ }^{39}$ and frustrations with continuing life-sustaining treatment where withdrawing treatment might be considered in the best interest of the patient when they have a life-threatening condition. ${ }^{23} 40$ Those findings indicate quite a contrast with opinions in this review where the focus was on management of advance decisions following suicidal behaviour and an expression of sympathy with the decision was not found. It will be important in future research to examine these differences further by contrasting views on management of advance decisions to refuse treatment following suicidal behaviour for patients with chronic and/or terminal physical conditions and patients without chronic or terminal physical conditions.

Management of the advance decision was difficult both emotionally and ethically for some clinicians because it challenged their professional training and their desire to protect vulnerable patients from suicide. The competing pressures of respecting a patient's right to autonomy while protecting them from the effects of mental disorder found in the current study is a commonly reported dilemma. ${ }^{41}$ There is evidence from the present study that support for the right to autonomy may be more dominant in clinicians from emergency medicine disciplines, with those from a psychiatric background prioritising prevention of suicide. A 'middle ground' between these views may help to provide guidance for clinicians. For example, in English law, courts have acknowledged that while some suicidal individuals may have capacity, the overwhelming likelihood is that capacity is impaired to at least some degree. ${ }^{41}$ Suicidal ideation has been associated with disordered and impulsive decision-making ${ }^{33} 34$ and evidence indicates that most mental health patients presenting to EDs are judged as not having capacity to make a treatment decision. ${ }^{12}$ Therefore a higher degree of certainty should be required when assessing capacity with suicidal behaviour and clinicians should err on the side of caution. ${ }^{8}$ Another potential resolution to this dilemma, particularly in emergency scenarios, may be to provide 'temporary intervention' to allow time for individuals to be assessed and treatment options to be discussed. ${ }^{41}$

An added pressure for clinicians in the management of advance decisions following suicidal behaviour was that they felt there was a societal expectation that suicide should be prevented. Adhering to the advance decision made by the patient by not treating them, not only was seen to go against their professional training to protect the patient, but it was viewed that this may be considered from a society perspective as unacceptable. The dilemma here is that a clinical decision of non-treatment and adherence with the advance decision might be accepted legally, but not socially. Concerns were expressed that this particular presentation of an advance decision met conditions that warranted overriding patients' autonomy because non-adherence with the advance decisions results in prevention of suicide, maintenance of the integrity of the medical professional and preservation of life. ${ }^{25}$

\section{Recommendations for practice}

Decisions made about advance decisions in the context of suicidal behaviour should be made in full consultation with psychiatric teams and with relevant legal and/ or ethical advisers. The results also highlight the importance of allocating sufficient time to consider contextual evidence relating to the suicidal behaviour, the authenticity of the treatment decision and verification of the documentation/decision. Given the gravity and emotive nature of a decision in this context, emergency healthcare workers may need increased support and supervision for such incidents.

Findings indicate that it may be helpful, in this particular context, for an advance decision to be written in consultation with a professional healthcare worker and the patient's family. This practice would also ensure that the patient is supported to consider all treatment options, that the advance decision is specific and detailed enough to be useful in an emergency situation and that patients' capacity at the time of writing the advance decision can be assessed and verified. The advance decision should be regularly reviewed and updated to ensure that it reflects the patient's current treatment decisions. 


\section{Strengths and limitations}

A strength of this review is that a broad range of articles from different disciplines were included, thus increasing the generalisability of results. However, there were some potential biases in the literature. First, there was a paucity of evidence: only six clinical cases were reported across the selected articles. There was also a risk of bias from the studies themselves, given that they were reviews of single clinical cases. Second, the articles were focused on the USA, UK and Australia, so may have resulted in bias relating to the specific legislation/ethics of those countries. There may be different views on this topic and its management in countries with different implementation of legislation, so it will be important for future research to compare findings internationally across a wider range of countries. ${ }^{42-44}$ Third, as with any syntheses of qualitative data there was potential for bias to be introduced by the research team at the stages of study identification, data extraction and synthesis. This was minimised in the current study by having two researchers carry out these tasks independently and cross-check the findings.

\section{Future directions}

Empirical studies, such as interviews and focus groups with clinicians and patients and/or a national clinical survey are important future priorities. Given that the presentation of an advance decision following suicidal behaviour is rare, case reports are likely to continue to be important sources of information in the future and authors should be mindful to ensure that case reports include details about how information about the case were obtained and how representative it is of other cases in this area. Research examining the prevalence of advance decisions relating to suicidal behaviour could shed light on the frequency of such presentations. Suitable platforms for storing advance decisions could also be explored. For example, some have suggested a web application ('app') could better reflect the dynamic nature of treatment refusal ${ }^{45}$ and make updating and reassessment easier.

\section{CONCLUSION}

Current literature on the management of advance decisions and suicidal behaviour centres on detailed accounts of clinical cases and demonstrates variability in practice and the rationale behind clinical decisions. Challenges in managing advance decisions specific to suicidal behaviour were evident, and there was some debate about whether advance decisions in the context of suicidal behaviour were appropriate in their current form. Taking time to consider all the evidence when making a decision, consulting fully with mental health clinicians and seeking legal and/or ethical advice may help with some of these challenges. The support of a relevant healthcare professional at the time of writing the advance decision may also be useful.

\section{Author affiliations}

${ }^{1}$ Centre for Mental Health and Safety, University of Manchester, Manchester, UK ${ }^{2}$ NHIR Greater Manchester Patient Safety Translational Research Centre, University of Manchester, UK

${ }^{3}$ Centre for Ethics in Medicine, University of Bristol, Bristol, UK

${ }^{4}$ Centre for Suicide Research, University Department of Psychiatry, Warneford Hospital, Oxford, UK

${ }^{5}$ Department of Population Health Sciences, University of Bristol, Bristol, UK

${ }^{6}$ School of Law, University of Manchester, Manchester, UK

${ }^{7}$ Emergency Department, Manchester Royal Infirmary, Manchester, UK

${ }^{8}$ Greater Manchester Mental Health NHS Foundation Trust, Chorlton House,

Manchester, UK

Acknowledgements We would like to thank Rosie Davies, Research Fellow in Patient and Public Involvement at the University of Bristol, for her input into the study.

Contributors All authors made substantial contributions to the study. RN and LQ conducted the initial scoping search. RN designed the review and data extraction/ analysis with input from NK and SS. RN and SS screened and reviewed the articles and performed data extraction/analysis, interpreted the results and wrote the first draft. NK, JC, RH, KH, DG, NA and KMJ reviewed the initial draft, contributed to subsequent drafts and approved the final version. All authors take responsibility for the integrity of the data analysis. NK is the guarantor of the study.

Funding This paper presents independent research funded by the National Institute of Health Research (NIHR) under its Programme Grants for Applied Research Programme (Grant Reference Number RP-PG-0610-10026).

Disclaimer The views expressed are those of the authors and not necessarily those of the NHS, the National Institute of Health Research or the Department of Health.

Competing interests DG, KH and NK are members of the Department of Health's (England) National Suicide Prevention Advisory Group. NK chaired the NICE guideline development group for the longer-term management of self-harm and the NICE Topic Expert Group (which developed the quality standards for self-harm services). He is currently chair of the updated NICE guideline for Depression. KH and DG are NIHR Senior Investigators. KH is also supported by the Oxford Health NHS Foundation Trust and NK by the Greater Manchester Mental Health NHS Foundation Trust.

Patient consent for publication Not required.

Provenance and peer review Not commissioned; externally peer reviewed. Data sharing statement № additional data are available.

Open access This is an open access article distributed in accordance with the Creative Commons Attribution 4.0 Unported (CC BY 4.0) license, which permits others to copy, redistribute, remix, transform and build upon this work for any purpose, provided the original work is properly cited, a link to the licence is given, and indication of whether changes were made. See: https://creativecommons.org/ licenses/by/4.0/.

\section{REFERENCES}

1. Department of Health. Mental capacity act. London: Stationery Office, 2005.

2. Byrne M. The use of advance directives. Nursing monograph. Melbourne: St. Vincent Healthcare Campus, 2002:13-16.

3. Campbell LA, Kisely SR. Advance treatment directives for people with severe mental illness. Cochrane Database Syst Rev 2009:CD005963.

4. Swanson JW, Swartz MS, Elbogen EB, et al. Facilitated psychiatric advance directives: a randomized trial of an intervention to foster advance treatment planning among persons with severe mental illness. Am J Psychiatry 2006;163:1943-51.

5. Morriss R, Mudigonda M, Bartlett P, et al. National survey and analysis of barriers to the utilisation of the 2005 mental capacity act by people with bipolar disorder in England and Wales. J Ment Health 2017;19:1-8. 
6. Dresser R. Suicide attempts and treatment refusals. Hastings Cent Rep 2010;40:10-11.

7. Frank C. How to reconcile advance care directives with attempted suicide. The Colorado Lawyer 2013;42:97-101.

8. Kapur N, Clements C, Bateman N, et al. Advance directives and suicidal behaviour. BMJ 2010;341:c4557-91.

9. Kapur N, Cooper J, O'Connor RC, et al. Non-suicidal self-injury v. attempted suicide: new diagnosis or false dichotomy? Br J Psychiatry 2013;202:326-8.

10. Hawton K, Bergen H, Casey D, et al. Self-harm in England: a tale of three cities. Soc Psychiatry Psychiatr Epidemiol 2007;42:513-21.

11. Carroll R, Metcalfe C, Gunnell D. Hospital presenting self-harm and risk of fatal and non-fatal repetition: systematic review and metaanalysis. PLoS One 2014;9:e89944.

12. Jacob R, Clare IC, Holland A, et al. Self-harm, capacity, and refusal of treatment: implications for emergency medical practice. A prospective observational study. Emerg Med J 2005;22:799-802.

13. Kapur N, Clements C, Bateman N, et al. Self-poisoning suicide deaths in England: could improved medical management contribute to suicide prevention? QJM 2010;103:765-75.

14. Brinkman-Stoppelenburg A, Rietjens JA, van der Heide A. The effects of advance care planning on end-of-life care: a systematic review. Palliat Med 2014;28:1000-25.

15. Houben CHM, Spruit MA, Groenen MTJ, et al. Efficacy of advance care planning: a systematic review and meta-analysis. J Am Med Dir Assoc 2014;15:477-89.

16. Moher D, Shamseer L, Clarke M, et al. PRISMA-P Group. Preferred reporting items for systematic review and meta-analysis protocols (PRISMA-P) 2015 statement. Syst Rev 2015;4:1.

17. Shamseer L, Moher D, Clarke M, et al. the PRISMA-P Group. Preferred reporting items for systematic review and meta-analysis protocols (PRISMA-P) 2015: elaboration and explanation. BMJ 2015;349:97647.

18. Centre for reviews and dissemination, Undertaking systematic reviews of research on effectiveness, CRD's guidance for carrying out or commissioning reviews. York: University of York, 2009.

19. Spencer L, Ritchie J. Qualitative data analysis for applied policy research. Analyzing qualitative data: Routledge, 2002:187-208.

20. Ryan R. Cochrane Consumers and Communication Review Group. Cochrane Consumers and Communication Review Group: data synthesis and analysis. $2013 \mathrm{http} / / /$ cccrg.cochrane.org (accessed 25th Apr 2018)

21. Murad MH, Sultan S, Haffar S, et al. Methodological quality and synthesis of case series and case reports. BMJ Evid Based Med 2018;23:60-3.

22. Mitchell M. An analysis of common arguments against Advance Directives. Nurs Ethics 2012;19:245-51.

23. Cook R, Pan P, Silverman R, et al. Do-not-resuscitate orders in suicidal patients: clinical, ethical, and legal dilemmas. Psychosomatics 2010;51:277-82.

24. Sontheimer D. Suicide by advance directive? J Med Ethics 2008;34:e4-e 1p.

25. Chalfin DB, Crippen D, Franklin C, et al. 'Round-table' ethical debate: is a suicide note an authoritative 'living will'? Crit Care 2001;5:115-24.
26. Callaghan S, Ryan CJ. Refusing medical treatment after attempted suicide: rethinking capacity and coercive treatment in light of the Kerrie Wooltorton case. J Law Med 2011;18:811-9.

27. David AS, Hotopf M, Moran P, et al. Mentally disordered or lacking capacity? Lessons for management of serious deliberate self harm. BMJ 2010;341:c4489.

28. Muzaffar S. 'To treat or not to treat'. Kerrie Wooltorton, lessons to learn. Emerg Med J 2011;28:741-4.

29. Richardson G. Mental capacity in the shadow of suicide: What can the law do? Int J Law Context 2013;9:87-105.

30. Ryan CJ, Callaghan S. Legal and ethical aspects of refusing medical treatment after a suicide attempt: the Wooltorton case in the Australian context. Med J Aust 2010;193:239-43.

31. Szawarski P. Classic cases revisited: the suicide of Kerrie Wooltorton $J$ Intensive Care Soc 2013;14:211-4.

32. Volpe RL, Levi BH, Blackall GF, et al. Exploring the limits of autonomy. Hastings Cent Rep 2012;42:16-18.

33. Jollant $F$, Bellivier $F$, Leboyer $M$, et al. Impaired decision making in suicide attempters. Am J Psychiatry 2005;162:304-10.

34. Dombrovski AY, Hallquist MN. The decision neuroscience perspective on suicidal behavior: evidence and hypotheses. Curr Opin Psychiatry 2017;30:7.

35. Baca-Garcia E, Diaz-Sastre C, García Resa E, et al. Suicide attempts and impulsivity. Eur Arch Psychiatry Clin Neurosci 2005;255:152-6.

36. Kleiman EM, Turner BJ, Fedor S, et al. Examination of real-time fluctuations in suicidal ideation and its risk factors: Results from two ecological momentary assessment studies. J Abnorm Psychol 2017;126:726-38.

37. Rosen DH. Suicide survivors. A follow-up study of persons who survived jumping from the Golden Gate and San Francisco-Oakland Bay Bridges. West J Med 1975;122:289.

38. Meisel A, Snyder L, Quill T. American College of Physicians-American Society of Internal Medicine End-of-Life Care Consensus Panel. Seven legal barriers to end-of-life care: myths, realities, and grains of truth. JAMA 2000;284:2495-501.

39. Scherer Y, Jezewski MA, Graves B, et al. Advance directives and end-of-life decision making: survey of critical care nurses' knowledge, attitude, and experience. Crit Care Nurse 2006;26:30-40.

40. Solomon MZ, O'Donnell L, Jennings B, et al. Decisions near the end of life: professional views on life-sustaining treatments. Am J Public Health 1993;83:14-23.

41. Allen $\mathrm{N}$. The right to life in a suicidal state. Int $\mathrm{J}$ Law Psychiatry 2013;36(5-6):350-7.

42. Blank RH. End-of-life decision making across cultures. J Law Med Ethics 2011;39:201-14.

43. Mishara BL, Weisstub DN. The legal status of suicide: a global review. Int J Law Psychiatry 2016;44:54-74.

44. van Wijmen MP, Rurup ML, Pasman HR, et al. Advance directives in the Netherlands: an empirical contribution to the exploration of a cross-cultural perspective on advance directives. Bioethics 2010;24:118-26.

45. Huxtable R. Advance decisions: worth the paper they are (not) written on? End of Life Journal 2015;5:e000002. 